The submitted manuscript has been authored by a contractor of the U.S.

\title{
COMMISSIONING OF THE LONG-PULSE FAST WAVE CURRENT DRIVE ANTENNAS FOR DII-D*
}

\author{
F. W. Baity, G. C. Barber, R. H. Goulding, D. J. Hoffman \\ Oak Ridge National Laboratory, Oak Ridge, Tennessee 37831-8071, USA \\ J. S. DeGrassie, R. I. Pinsker, C. C. Petty, W. Cary \\ General Atomics, San Diego, CA 92186-9784
}

\section{INTRODUCTION}

Two new four-element fast wave current drive antennas 1 have been installed on DIII-D. These antennas are designed for $10-\mathrm{s}$ pulses at $2 \mathrm{MW}$ each in the frequency range of 30 to $120 \mathrm{MHz}$. Each element comprises two poloidal segments fed in parallel in order to optimize plasma coupling at the upper end of the frequency range. The antennas are mounted on opposite sides of the vacuum vessel, in ports designated $0^{\circ}$ and $180^{\circ}$ after their toroidal angle.

Each antenna array is fed by a single transmitter. The power is first split two ways by means of a 3-dB hybrid coupler, then each of these lines feeds a resonant loop connecting a pair of array elements. The power transfer during asymmetric phasing is shunted between resonant loops by a decoupler. The resonant loops are fitted with line stretchers so that multiple frequencies of operation are possible without reconfiguring the transmission line.

Commissioning of these antennas has been underway since June, 1994. Several deficiencies in the transmission line system were uncovered during initial vacuum conditioning, including problems with the transmission line insulators and with the drive rods for the variable elements. The former was solved by replacing the original alumina insulators, and the latter has been avoided during operation to date by positioning the tuners to avoid high voltage appearing on the drive rods. A modified design for the drive rods will be implemented before RF operations resume operation in June, 1995.

New transmitters were procured from $\mathrm{ABB}$ for the new antennas and were installed in parallel with the antenna installation. The transmitter commissioning was hampered by a number of difficulties, including a failure of a high voltage transformer and three FPA tube failures. However, both transmitters were in operation at reduced power for the February run.

During initial vacuum conditioning of the antenna in the $180^{\circ}$ port a fast digital oscilloscope was used to try to pinpoint the location of arcing by a time-of-flight technique and to develop an understanding of the typical arc signature in the system. This is viewed as the first step in the development of a possible ELM discrimination method for later implementation.

\section{INITIAL OPERATION WITH THE $0^{\circ}$ ANTENNA}

All components of the system were brand new, so the initial vacuum conditioning efforts have been primarily a shakedown for the transmitters, transmission lines, and antennas. A schematic of the transmission line system is shown in Fig. 1. The initial intention was to perform preliminary checkout and conditioning in symmetric (heating) phasing in order to simplify the tuning and matching; however, the reflected signals caused oscillation problems in the transmitters when connected to the antenna load. Hence, current drive phasing was used in order to eliminate antenna reflections at the transmitter. When the two output feed lines from the hybrid splitter are $90^{\circ}$ out of phase, the reflected power is predominately directed into the dummy load and away from the transmitter. The incorporation of the decoupler into the circuit has nevertheless simplified the task of impedance matching to that of matching a single uncoupled transmission line. The decoupler,

*Research sponsored by the Office of Fusion Energy, U.S. Department of Energy, under contract DE-AC0584OR21400 with Martin Marietta Energy Systems, Inc. and contract DE-AC03-89ER51114 with General Atomics 


\section{DISCLAIMER}

Portions of this document may be illegible in electronic image products. Images are produced from the best available original document. 


\section{Commissioning Of The Long-Pulse Fast Wave Current Drive Antennas For DIII-D}

susceptance null stubs, and resonant loop line stretchers were preset for vacuum loading resistance at the chosen operating frequency with a network analyzer. The decoupler setting is a function of plasma loading, but using the vacuum setting does not create a large imbalance between lines for low to moderate plasma loading.

The $0^{\circ}$ antenna system was available for operation beginning in July, 1994 . The $180^{\circ}$ system was first operated in February, 1995. To date most of the difficulties encountered have been attributed to the transmitters and transmission line. Modifications have been made where appropriate to improve the voltage handling of the transmission line to beyond the designed operating limit of $30 \mathrm{kV}$. Given the problems in the transmission line, we are not yet in a position to compare the effectiveness of nickel plating over copper plating of the antennas for reducing the time required for conditioning.

During initial vacuum conditioning the maximum voltage in the $26-\Omega$ resonant loops was increased rapidly to a peak value of $13 \mathrm{kV}$, but then deteriorated to $10 \mathrm{kV}$, accompanied by audible arcing in one of the resonant loops. All transmission line in the system uses three alumina rods for support of the inner conductor. Several burned polyethylene insulator pads at the end of the alumina rods were found in this section of line. These pads were affixed by the manufacturer for the purpose of facilitating the insertion of the inner conductor into the outer conductor; however, as a result of this failure, all such insulator pads were removed from the untuned portions of transmission line. Subsequently all alumina insulators were replaced with lower dielectric constant material having fully radiused and polished surfaces. Tests at General Atomics and ORNL have showî the redesigned insulators to have superior voltage handling capabilityicorympared to the original alumina supports. No further problems have been experienced with the transmission line insulators.

Thus modified, the $0^{\circ}$ antenna was operated in plasma at a power level of $1 \mathrm{MW}$, limited by available transmitter output at the time. However, during long-pulse vacuum conditioning a fiberglass stub tuner push rod arced over at a voltage of $25-30 \mathrm{kV}$. The push rod failure occurred in gradual but steady fashion, suggesting that the failure was due to overheating of the fiberglass from dielectric losses, possibly enhanced by water absorption. The worst damage was observed at the end of the rod where it attached to the center conductor of the stub. This failure was also located audibly. The damaged tuner was temporarily replaced with a fixed stub, and the remaining tunable stubs were reset to a position a half wavelength longer so that the voltage at the push rod was near a minimum and so that the length along the insulator surface was increased. All the tuners are being reworked with corona rings and Delrin push rods, which have shown excellent voltage handling capability.

\section{VACUUM CONDITIONING OF THE $180^{\circ}$ ANTENNA}

The $180^{\circ}$ antenna has been installed in the DIII-D vacuum vessel since February, 1994. The first vacuum conditioning with RF power was attempted in February, 1995, at which time the antenna had been through numerous bake cycles and a year of DIII-D plasma operations without RF. Nevertheless there was initially substantial gas evolution from the antenna, which diminished steadily over the first several hours of pulsed conditioning. The voltage on the antenna was pushed to $16 \mathrm{kV}$ in about 30 minutes of short pulse operation. The pulses were lengthened to $2 \mathrm{~s}$, at which time there were occasional large gas bursts associated with transmitter trips. Plasma operation during this period was hampered by the lack of conditioning time and by the arcing in the transmission line discussed below.

When the voltage reached $20 \mathrm{kV}$, an arc occurred that reduced the voltage handling by a factor of 5. The system gradually recovered its previous level over the next hour, but this event was repeated several times in the next few days. This arc was localized to the section of transmission 


\section{Commissioning Of The Long-Pulse Fast Wave Current Drive Antennas For DIII-D}

line between the single stub tuner and the resonant loop tee by digitizing the breakdown as described in the next section. DC hi-pot testing was then used to pinpoint the actual location in this section, which is about $60 \mathrm{~m}$ long. A badly charred o-ring was found at this location. The dc voltage required for breakdown was $38 \mathrm{kV}$, nearly double the maximum peak RF voltage attained.

\section{SEPARATING ARCS FROM ELMS}

The problem of discriminating between transmission line arcs and ELMs needs to be addressed for successful ICRF heating of H-mode discharges. Both arcs and ELMs can cause large increases in reflected power on a time scale much too fast for mechanical compensation in external tuning elements. Antennas handling megawatts of RF power require rapid removal of this power in the event of a transmission line arc in order to prevent serious damage to the affected component. On the other hand, it is desirable to try to maintain as much RF power as possible during ELMs. In addition, with increasingly long transmission lines between the transmitters and antennas, a method of localizing arcs occurring in the transmission line is needed. Little data exist on the time history of ELMs and their effect on antenna performance.

One possible method for distinguishing arcs from ELMs for typical antennas is from the rise time of reflected power. The typical rise time for ELMs in DIII-D is on the order of milliseconds, whereas arcs are expected to occur much faster. The DIII-D transmission line systems have directional couplers at several locations, and there are current and voltage probes on the antennas. During vacuum conditioning of the $180^{\circ}$ system, measurements were made using digitizer sampling rates up to $200 \mathrm{MHz}$, with memory sufficient to store up to 25 arc events during a single rf pulse into four data channels. RF reflected voltage signals from directional couplers located in the two external resonant loops, on the unmatched side of the lines feeding these loops, and in the line leading from the hybrid power splitter to the dummy load were recorded. Two distinct arc signatures were found: fast arcs with rise times less than $1 \mu \mathrm{s}$, and slow "arcs" developing over tens of milliseconds. A comparison is shown in Fig. 2.

For the fast arcs a time-of-flight measurement could, in principle, determine the approximate location of the arc, at least for vacuum conditioning. Our first attempt to locate arcs by the time-offlight technique during plasma operation was inconclusive, due to the increased noise on the reflected power signals from the plasma edge fluctuations. Reflected power signals are a good choice for this technique in the impedance-matched sections of transmission line. The difficulty with using directional coupler signals in the untuned line is that the reflected power is normally high, and an arc may not change the magnitude by a sufficient amount. A voltage probe located near a node in the voltage waveform should be a better choice for the untuned transmission line.

\section{PRESENT STATUS}

Both new fast wave current drive systems are in operation on DIII-D. Problems with the transmission line have been eliminated as encountered, and both transmission, line systems should be operating at design specification for the next run period. There has been one day of simultaneous operation of all three FWCD systems. A typical shot from this run is shown in Fig. 3. The main impediment to full power operation is now the transmitters, which have not yet passed the final acceptance testing.

1. F. W. Baity et al, "Design of Long-Pulse Fast Wave Current Drive Antennas for DIII-D," AIP Conf. Proc. 289, 343 (Boston, MA 1993).

May 5, 1995 


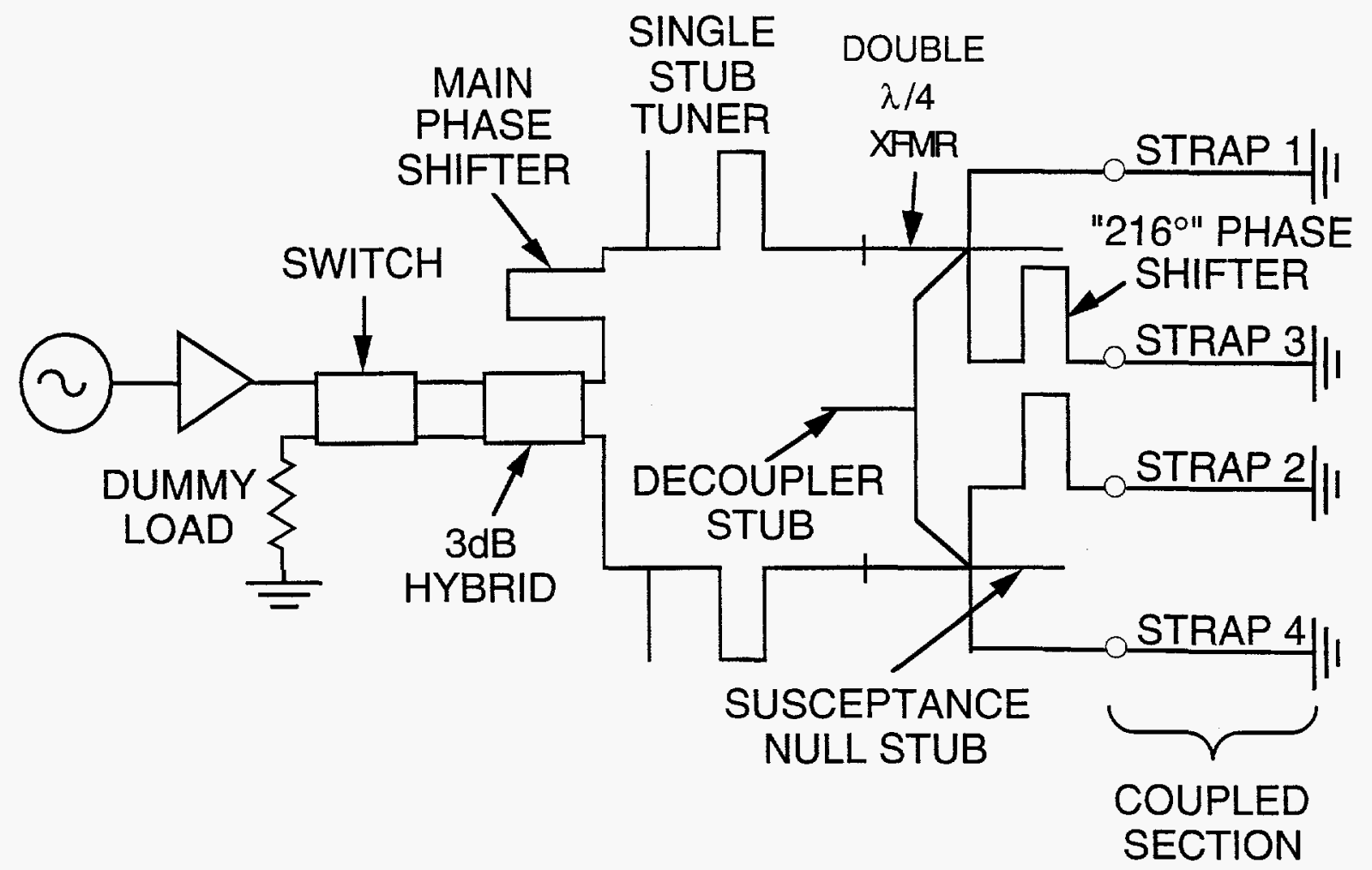

Fig. 1. Schematic of the long-pulse FWCD antennas on DIII-D. The resonant loops connecting pairs of antenna straps have a $26-\Omega$ characteristic impedance. All other transmission line sections are $50-\Omega$.

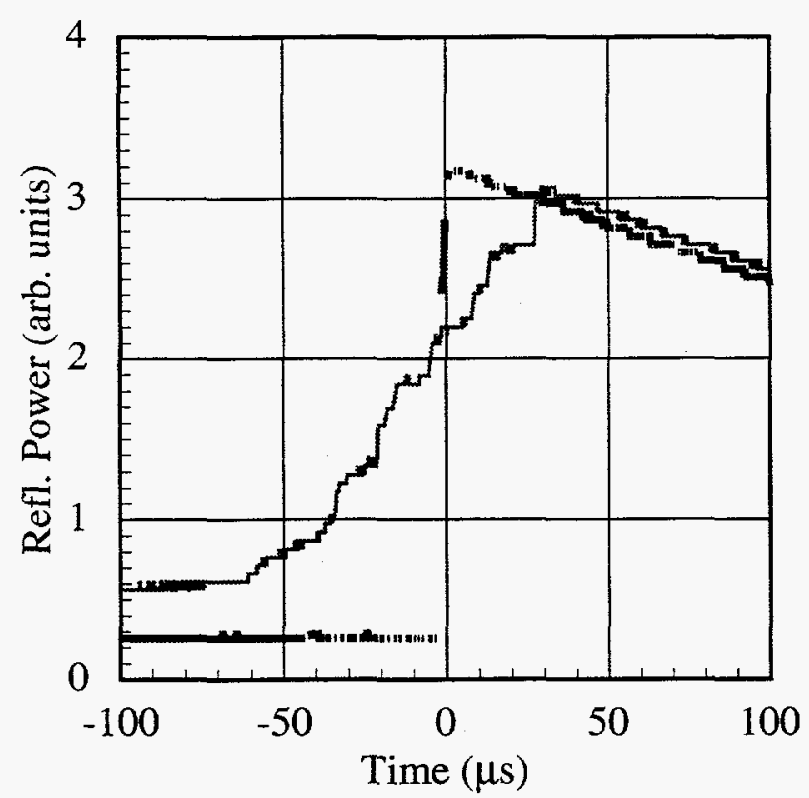

Fig. 2. Examples of slow and fast arcs seen during vacuum conditioning of the $180^{\circ}$ antenna system. Plotted is the power into the dummy load as a result of the arc.

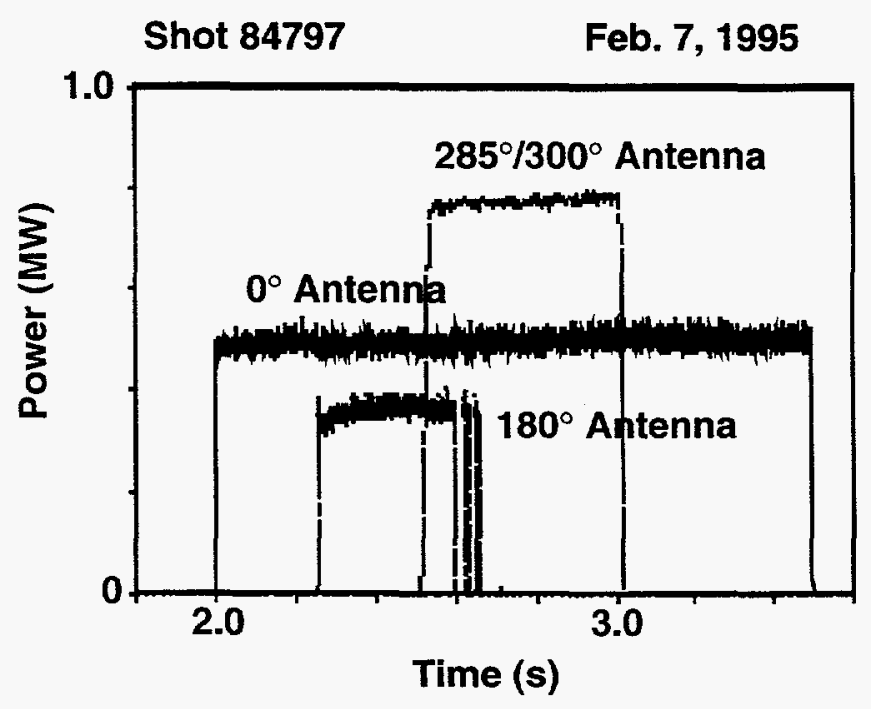

Fig. 3. All three FWCD systems operated simultaneously. The $285^{\circ} / 300^{\circ}$ system was tuned to $60 \mathrm{MHz}$, the $0^{\circ}$ system to $90.1 \mathrm{MHz}$, and the $180^{\circ}$ system to $93.84 \mathrm{MHz}$. 